\title{
A NOTE ON BLOCK SEQUENCES IN HILBERT SPACES
}

\author{
S. K. Kaushik, Ghanshyam Singh and Virender \\ University of Delhi and M.L.S. University, India
}

\begin{abstract}
Block sequences with respect to frames in Hilbert spaces have been defined. Examples have been provided to show that a block sequence with respect to a given frame may not even be a Bessel sequence. Also, a necessary and sufficient condition under which a block sequence with respect to a frame is a frame has been given. Further, applications of block sequences to obtain Fusion frames and Fusion frame systems have been given. Finally, a problem has been posed and observed that an affirmative answer to this problem gives an affirmative answer to the Feichtinger Conjecture.
\end{abstract}

\section{INTRODUCTION}

In 1952, Duffin and Schaeffer [13] introduced frames for Hilbert spaces. It took more than 30 years to realize the importance of frames. But, after the landmark paper of Daubechies, Grossmann and Meyer [12], in 1986, the theory of frame began to be more widely studied. For an introduction to frames, one may refer to $[3,10,11,14,15,16]$. Casazza [4], and Benedetto and Fickus [2] have studied frames in finite dimensional spaces which attracted more attention due to their use in signal and image processing. For signal reconstruction without phase information, one may refer to [1]. Frames, now a days, are main tools for use in signal and image processing, data compression, sampling theory, optics, filter banks, signal detection, time frequency analysis etc.

A number of new applications have emerged which cannot be modeled naturally by one single frame system. In such cases, the data assigned to one single frame system becomes too large to be handled numerically. So, it would be beneficial to split large frame system into a set of much smaller systems

2000 Mathematics Subject Classification. 42C15, 42A38.

Key words and phrases. Frame, Bessel sequence, block sequence. 
and to process the data locally within each subsystem effectively. Thus, a distributed frame theory for a set of local frame systems is required. In this direction, a theory based on Fusion frames was developed in $[6,8,9,18]$ which provides a framework to deal with these applications and to derive efficient and robust algorithms.

Another well known requirement in the frame theory is to decompose a bounded frame into finite union of Riesz basic sequences. This is known as Feichtinger conjecture and is connected to the famous Kadison-Singer conjecture [17]. It was shown in [5] that Kadison-Singer conjecture implies Feichtinger conjecture. For more results regarding the Feichtinger conjecture, one may refer to $[5,7]$.

In the present paper, we define block sequences is Hilbert spaces and give examples to show that a block sequence with respect to a given frame need not be a frame (frame sequence). Also, a necessary and sufficient condition under which a block sequence with respect to a given frame is a frame has been given. Further, we discuss applications of block sequences to obtain fusion frames and fusion frame systems. Finally, we pose a problem and observe that an affirmative answer to this problem gives an affirmative answer to the Feichtinger conjecture.

\section{Preliminaries}

Throughout the paper, $H$ will denote an infinite dimensional Hilbert space, $\left\{n_{k}\right\}$ an infinite increasing sequence in $\mathbb{N},\left[x_{n}\right]$ the closed linear span of $\left\{x_{n}\right\}$, for any set $D, O(D)$ will denote the cardinality of $D$.

Definition 2.1. A sequence $\left\{x_{n}\right\} \subset H$ is called a frame for $H$ if there exist constants $A, B>0$ such that

$$
A\|x\|^{2} \leq \sum_{n \in \mathbb{N}}\left|\left\langle x, x_{n}\right\rangle\right|^{2} \leq B\|x\|^{2}, \quad x \in H
$$

The positive constants $A$ and $B$, respectively, are called lower and upper frame bounds of the frame $\left\{x_{n}\right\}$. The inequality (2.1) is called the frame inequality.

The frame $\left\{x_{n}\right\} \subset H$ is called tight if it is possible to choose $A, B$ satisfying inequality (2.1) with $A=B$ and is called normalized tight if $A=B=1$. The frame $\left\{x_{n}\right\} \subset H$ is called exact if removal of any arbitrary $x_{n}$ renders the collection $\left\{x_{n}\right\}$ no longer a frame for $H$. A sequence $\left\{x_{n}\right\} \subset H$ is called a Bessel sequence if it satisfies upper frame inequality in (2.1). A sequence $\left\{x_{n}\right\} \subset H$ is said to be a frame sequence for $H$ if $\left\{x_{n}\right\}$ is a frame for $\left[x_{n}\right]$. 


\section{Main Results}

Definition 3.1. A sequence $\left\{y_{n}\right\}$ in a Hilbert space $H$ is said to be a block sequence with respect to a sequence $\left\{x_{n}\right\}$ in $H$ if it is of the form

$$
y_{n}=\sum_{i \in D_{n}} \alpha_{i} x_{i} \neq 0, \quad n \in \mathbb{N}
$$

where $D_{n}$ 's are finite subsets of $\mathbb{N}$ with $D_{n} \cap D_{m}=\phi, n \neq m, \bigcup_{n \in \mathbb{N}} D_{n}=\mathbb{N}$ and $\alpha_{i}$ 's are any scalars.

The following observations arise naturally in wake of the block sequences with respect to frames.

\section{OBSERVATIONS}

(I) A block sequence with respect to a frame in a Hilbert space may or may not be a frame for $H$.

Indeed:

(a) Let $\left\{x_{n}\right\}$ be any frame for $H$, then for scalars $\alpha_{i}=1, i \in \mathbb{N}$ and $D_{n}=\{n\}, n \in \mathbb{N},\left\{y_{n}\right\}$ (as given in (3.1)) is a frame for $H$.

(b) Let $\left\{x_{n}\right\}$ be a sequence of orthonormal unit vectors in $H$. Then $\left\{x_{n}\right\}$ is a frame for $H$. Take $\alpha_{i}=1, i \in \mathbb{N}$ and $D_{1}=\{1,2,3\}$; $D_{n}=\{2+n\}$, for all $n \geq 2$. Then the block sequence $\left\{y_{n}\right\}$ is a frame sequence for $H$ but it is not a frame for $H$.

(II) A block sequence with respect to a frame in $H$ may not even be a frame sequence for $H$. Indeed, let $\left\{e_{n}\right\}$ be the sequence of orthonormal unit vectors in $H$ and $\left\{x_{n}\right\}$ in $H$ be defined by

$$
x_{1}=e_{1} ; x_{2 n}=x_{2 n+1}=e_{n+1}, \quad \text { for all } n \geq 1 .
$$

Then $\left\{x_{n}\right\}$ is a frame for $H$. Take $\alpha_{i}=1, i \in \mathbb{N}$ and $D_{1}=\{1\}$; $D_{n}=\{2 n-2,2 n-1\}$, for all $n \geq 2$. Then the block sequence $\left\{y_{n}\right\}$ is not a frame sequence for $H$. However, $\left[y_{n}\right]=H$.

(III) A block sequence with respect to a sequence in $H$ which is not even a frame for $H$ may be a frame for $H$. Indeed, let $\left\{e_{n}\right\}$ be a sequence of orthonormal unit vectors in $H$. Define $\left\{x_{n}\right\}$ in $H$ by $x_{n}=\frac{e_{n}}{n}, n \in \mathbb{N}$. Then $\left\{x_{n}\right\}$ is not a frame for $H$. Take $\alpha_{n}=n, n \in \mathbb{N}$ and $D_{n}=\{n\}$, $n \in \mathbb{N}$. Then the block sequence $\left\{y_{n}\right\}$ with respect to $\left\{x_{n}\right\}$ is a frame for $H$.

(IV) A block sequence $\left\{y_{n}\right\}$ with respect to a frame $\left\{x_{n}\right\}$ in $H$ with $\inf \left\|y_{n}\right\|>\delta$, for all $n \in \mathbb{N}$, for some $\delta>0$, may fail to ensure that $\left\{y_{n}\right\}$ is a frame sequence for $H$ (see Observation (II)).

(V) A block sequence $\left\{y_{n}\right\}$ with respect to a frame $\left\{x_{n}\right\}$ with $\left[y_{n}\right]=\left[x_{n}\right]$ may also fail to ensure that $\left\{y_{n}\right\}$ is a frame sequence for $H$. Indeed, let $\left\{x_{n}\right\}$ be the sequence of orthonormal unit vectors in $H$. Take $\alpha_{i}=\frac{1}{i}$, 
$i \in \mathbb{N}$ and $D_{n}=\{n\}, n \in \mathbb{N}$. Then $\left[y_{n}\right]=\left[x_{n}\right]$. But $\left\{y_{n}\right\}$ is not a frame sequence for $H$.

In view of the above observations, one may ask for sufficient conditions under which a block sequence with respect to a frame is a Bessel sequence (frame) for $H$. We prove the following result in this direction.

ThEOREM 3.2. Let $\left\{x_{n}\right\}$ be a frame for $H$ and $\left\{y_{n}\right\}$ be a block sequence with respect to $\left\{x_{n}\right\}$. Then $\left\{y_{n}\right\}$ is a frame for $H$ if $\sup _{1 \leq i<\infty}\left|\alpha_{i}\right|<\infty$, $\sup \left\{O\left(D_{n}\right): n \in \mathbb{N}\right\}<\infty$ and

$$
\inf _{n}\left[\sum_{i \in D_{n}}\left|\alpha_{i}\right|^{2}-\sum_{\substack{i, j \in D_{n} \\ i \neq j}}\left|\alpha_{i} \bar{\alpha}_{j}\right|\right]>0 .
$$

Further, if $\left\{x_{n}\right\}$ is exact, then $\left\{y_{n}\right\}$ is also exact.

Proof. Let $0<A \leq B<\infty$ be constants such that

$$
A\|x\|^{2} \leq \sum_{n}\left|\left\langle x_{n}, x\right\rangle\right|^{2} \leq B\|x\|^{2}, \quad x \in H .
$$

Now

$$
\begin{aligned}
\sum_{n}\left|\left\langle y_{n}, x\right\rangle\right|^{2}= & \sum_{n}\left|\left\langle\sum_{i \in D_{n}} \alpha_{i} x_{i}, x\right\rangle\right|^{2} \\
\leq & \sup _{1 \leq i<\infty}\left|\alpha_{i}\right|^{2} \sum_{n} K_{0}\left(\sum_{i \in D_{n}}\left|\left\langle x_{n}, x\right\rangle\right|^{2}\right), \\
& \left(\text { where } K_{0}=\sup _{n}\left\{k_{n}: k_{n}=O\left(D_{n}\right), \text { cardinality of } D_{n}\right\}\right) \\
\leq & K_{0} \sup _{1 \leq i<\infty}\left|\alpha_{i}\right|^{2} B\|x\|^{2}, \quad x \in H .
\end{aligned}
$$

Therefore, $\left\{x_{n}\right\}$ is a Bessel sequence for $H$ with bound $K_{0} \sup _{1 \leq i<\infty}\left|\alpha_{i}\right|^{2} B=B_{0}$.

Again

$$
\begin{aligned}
\sum_{n}\left|\left\langle y_{n}, x\right\rangle\right|^{2} & =\sum_{n}\left|\left\langle\sum_{i \in D_{n}} \alpha_{i} x_{i}, x\right\rangle\right|^{2} \\
& =\sum_{n} \sum_{i \in D_{n}}\left|\alpha_{i}\right|^{2}\left|\left\langle x_{i}, x\right\rangle\right|^{2}+\sum_{n} \sum_{\substack{i, j \in D_{n} \\
i \neq j}} \alpha_{i} \bar{\alpha}_{j}\left\langle x_{i}, x\right\rangle\left\langle x, x_{j}\right\rangle \\
& \geq \sum_{n} \sum_{i \in D_{n}}\left|\alpha_{i}\right|^{2}\left|\left\langle x_{i}, x\right\rangle\right|^{2}-\sum_{n} \sum_{i \in D_{n}}\left|\left\langle x_{n}, x\right\rangle\right|^{2} \sum_{\substack{i, j \in D_{n} \\
i \neq j}}\left|\alpha_{i} \bar{\alpha}_{j}\right| .
\end{aligned}
$$


Therefore

$$
\sum_{n}\left|\left\langle y_{n}, x\right\rangle\right|^{2} \geq A_{0}\|x\|^{2}, \quad x \in E
$$

where $A_{0}=a A$ and $a=\inf _{n}\left[\sum_{i \in D_{n}}\left|\alpha_{i}\right|^{2}-\sum_{\substack{i, j \in D_{n} \\ i \neq j}}\left|\alpha_{i} \bar{\alpha}_{j}\right|\right]$.

Hence $\left\{y_{n}\right\}$ is a frame for $H$ with bounds $A_{0}$ and $B_{0}$.

Further, if $\left\{y_{n}\right\}$ is not exact, then, for some $m \in \mathbb{N}$

$$
y_{m}=\sum_{j \neq m} \beta_{j} y_{j}
$$

where $\beta_{j} \neq 0$ for some $j \neq m$. This gives

$$
\sum_{i \in D_{m}} \alpha_{i} x_{i}=\sum_{j \neq m} \beta_{j}\left(\sum_{k \in D_{j}} \alpha_{k} x_{k}\right)
$$

where $\alpha_{i_{0}} \neq 0$ for some $i_{0} \in D_{m}$. Therefore, for some $c_{n} \neq 0, n \neq i_{0}$, we may write $x_{i_{0}}=\sum_{n \neq i_{0}} c_{n} x_{n}$. Hence $\left\{x_{n}\right\}$ is not exact.

REMARK 3.3. (i) The condition $\sup \left\{O\left(D_{n}\right): n \in \mathbb{N}\right\}<\infty$ can not be dropped as if we consider $H=\ell_{2}$ with orthonormal basis $\left\{e_{n}\right\}$ and let $D_{n}=\left\{\frac{1}{2} n(n-1)+k: k=1,2, \ldots, n\right\}, n \in \mathbb{N}$. Clearly $D_{n} \cap D_{m}=\phi$ for $n \neq m$ and $\bigcup_{n \in \mathbb{N}} D_{n}=\mathbb{N}$. Also note that $\sup \left\{O\left(D_{n}\right): n \in \mathbb{N}\right\} \nless \infty$. Let $\left\{y_{n}\right\}$ be a block sequence defined by the relation

$$
y_{n}=\sum_{i \in D_{n}} \alpha_{i} e_{i}, \text { where } \alpha_{i}=1 \text { for all } i \in \mathbb{N} .
$$

Then, for $x=\sum_{n} n^{-3 / 2} \sum_{i \in D_{n}} e_{i}$ in $H,\|x\|<2$, but $\sum\left|\left\langle x, y_{n}\right\rangle\right|^{2}$ does not converge and hence $\left\{y_{n}\right\}$ is not a Bessel sequence, so can not be a frame for $H$.

(ii) In Theorem 3.2, the condition that

$$
\inf _{n}\left[\sum_{i \in D_{n}}\left|\alpha_{i}\right|^{2}-\sum_{\substack{i, j \in D_{n} \\ i \neq j}}\left|\alpha_{i} \bar{\alpha}_{j}\right|\right]>0
$$

is not necessary (see the details of Observation $\mathrm{I}(\mathrm{b})$ ). Also, we can not drop this condition as the block sequence $\left\{y_{n}\right\}$ in Observation (V) is 
not a frame and in this case

$$
\inf _{n}\left[\sum_{i \in D_{n}}\left|\alpha_{i}\right|^{2}-\sum_{\substack{i, j \in D_{n} \\ i \neq j}}\left|\alpha_{i} \bar{\alpha}_{j}\right|\right] \ngtr 0
$$

(iii) The condition that $\sup _{1 \leq n<\infty}\left|\alpha_{n}\right|<\infty$ is not necessary. Indeed, let $\left\{e_{n}\right\}$ is a sequence of orthonormal unit vectors and $\left\{x_{n}\right\}$ is defined as

$$
x_{2 n-1}=e_{n} \quad \text { and } \quad x_{2 n}=\frac{e_{n}}{n}, \quad n \in \mathbb{N} .
$$

Then the block sequence $\left\{y_{n}\right\}$ defined by $y_{n}=x_{2 n-1}+n x_{2 n}, n \in \mathbb{N}$ is also a frame for $H$, but $\sup \left|\alpha_{i}\right| \nless \infty$.

We now give a necessary and sufficient condition under which a block sequence with respect to a frame is a frame.

THEOREM 3.4. Let $\left\{x_{n}\right\}$ be a frame for $H$ and $\left\{y_{n}\right\}$ be a block sequence with respect to $\left\{x_{n}\right\}$. Let $T: \ell_{2}(\mathbb{N}) \rightarrow \ell_{2}(\mathbb{N})$ be a bounded linear operator such that $T\left(\left\{\left\langle x_{n}, x\right\rangle\right\}\right)=\left\{\left\langle y_{n}, x\right\rangle\right\}, x \in H$. Then $\left\{y_{n}\right\}$ is also a frame for $H$ if and only if there exists a $\lambda>0$ such that

$$
\left\|\left\{\left\langle y_{n}, x\right\rangle\right\}\right\| \geq \lambda\left\|\left\{\left\langle x_{n}, x\right\rangle\right\}\right\|, \quad x \in H .
$$

Proof. Let $0<A \leq B<\infty$ be such that

$$
A\|x\|^{2} \leq \sum_{n}\left|\left\langle x_{n}, x\right\rangle\right|^{2} \leq B\|x\|^{2}, \quad x \in H .
$$

Then

$$
\sum_{n}\left|\left\langle y_{n}, x\right\rangle\right|^{2}=\left\|\left\{\left\langle y_{n}, x\right\rangle\right\}\right\|^{2} \geq \lambda^{2} A\|x\|^{2}, \quad x \in H
$$

Also

$$
\sum_{n}\left|\left\langle y_{n}, x\right\rangle\right|^{2}=\left\|\left\{\left\langle y_{n}, x\right\rangle\right\}\right\|^{2}=\left\|T\left(\left\{\left\langle x_{n}, x\right\rangle\right\}\right)\right\|^{2} \leq\|T\|^{2} B\|x\|^{2}, \quad x \in H .
$$

Hence $\left\{y_{n}\right\}$ is frame for $H$ with bounds $\lambda^{2} A$ and $\|T\|^{2} B$.

Conversely, let $\left\{y_{n}\right\}$ is a frame for $H$ with bounds $A_{y}$ and $B_{y}$.

Then, for any $x \in H$, we have

$$
A_{y}\|x\|^{2} \leq \sum_{n}\left|\left\langle y_{n}, x\right\rangle\right|^{2} \leq B_{y}\|x\|^{2}, \quad x \in H .
$$

Therefore, by (3.2)

$$
\frac{A_{y}}{B} \sum_{n}\left|\left\langle x_{n}, x\right\rangle\right|^{2} \leq A_{y}\|x\|^{2} \leq \sum_{n}\left|\left\langle y_{n}, x\right\rangle\right|^{2}, \quad x \in H .
$$


Put $\frac{A_{y}}{B}=\lambda^{2}$. Then $\lambda>0$ such that

$$
\left\|\left\{\left\langle y_{n}, x\right\rangle\right\}\right\| \geq \lambda\left\|\left\{\left\langle x_{n}, x\right\rangle\right\}\right\|, \quad x \in H .
$$

\section{ApPLICATIONS}

Let $\left\{x_{n}\right\}$ be a frame for $H$ and let $V_{n}=\left[x_{i}\right]_{i \in D_{n}}, n \in \mathbb{N}$. Then $\left\{V_{n}\right\}$ is a sequence of subspaces of $H$ such that $\bigcup_{n} V_{n}=H$. Therefore, each $x \in H$ can be expressed as

$$
x=\sum_{i=1}^{\infty} y_{i}
$$

where $y_{i} \in V_{i}, i \in \mathbb{N}$. The representation in (3.3) may not be unique. Note that, for each $n \in \mathbb{N},\left\{x_{i}\right\}_{i \in D_{n}}$ is a frame for the subspace $V_{n}$. Define $v_{n}: H \rightarrow V_{n}$ by

$$
v_{n}(x)=\sum_{i \in D_{n}} \alpha_{i} x_{i} \in V_{n}, n \in \mathbb{N}, x \in H .
$$

Then one can find constants $0<A \leq B<\infty$ such that

$$
A\|x\|^{2} \leq \sum_{i=1}^{\infty}\left\|v_{i}(x)\right\|^{2} \leq B\|x\|^{2}, \quad x \in H .
$$

Therefore $\left(V_{n}, v_{n}\right)_{n \in \mathbb{N}}$ is a Fusion frame for $H$ and $\left(V_{n}, v_{n},\left\{x_{i}\right\}_{i \in D_{n}}\right)$ is a Fusion frame system for $H$ (Fusion frames and Fusion frame systems were introduced and studied recently by Casazza and Kutyniok and others in $[6$, $8,9,18])$.

If $\left\{x_{n}\right\}$ is exact frame for $H$, then there exists a sequence of finite subsets $\left\{D_{n}\right\}$ of $\mathbb{N}$ (e.g. take $D_{n}=\{n\}, n \in \mathbb{N}$ ) with $\bigcup_{n \in \mathbb{N}} D_{n}=\mathbb{N}$ such that $H=$ $\bigoplus_{n \in \mathbb{N}} V_{n}$, where $V_{n}=\left[x_{i}\right]_{i \in D_{n}}$.

Also, $\left\{f_{n}\right\}$ be the sequence of unit vectors in $H$. Define a sequence $\left\{g_{n}\right\}$ in $H$ by $g_{n}=\frac{1}{\sqrt{n}} f_{n}, n \in \mathbb{N}$. Let $n_{k}=n_{k-1}+(k-1), k \in \mathbb{N}$ and $n_{0}=1$. Then $\left\{n_{k}\right\}$ is an infinite increasing sequence in $\mathbb{N}$. Now define $\left\{h_{n}\right\}$ in $H$ by

$$
h_{1}=g_{1}, h_{n_{k}}=h_{n_{k}+1}=h_{n_{k}+2}=\cdots=h_{n_{k+1}-1}=g_{k}, k \geq 2 .
$$

Then $\left\{h_{n}\right\}$ is a tight non-exact frame for $H$. Taking $D_{k}=\left\{n_{k}, n_{k}+1\right.$, $\left.\ldots, n_{k+1}-1\right\}, k \in \mathbb{N}$ and $V_{k}=\left[h_{i}\right]_{i \in D_{k}}, k \in \mathbb{N}$, we get $H=\bigoplus_{n \in \mathbb{N}} V_{n}$.

In view of the above discussion, it is natural to raise the following problem. 
Problem 3.1. Is it always possible to have a sequence of finite subsets $\left\{D_{n}\right\}$ of $\mathbb{N}$ with $\bigcup_{n} D_{n}=\mathbb{N}$ such that $H=\bigoplus_{n \in \mathbb{N}} V_{n}$, where $V_{n}=\left[x_{i}\right]_{i \in D_{n}}$ and $\left\{x_{n}\right\}$ is a bounded frame for $H$ ?

REMARK 3.5. An affirmative answer to this problem solves the Feichtinger conjecture in affirmative. Indeed, let $\left\{D_{n}\right\}_{n \in \mathbb{N}}$ be a sequence of finite subsets of $\mathbb{N}$ with $\bigcup_{n \in \mathbb{N}} D_{n}=\mathbb{N}$ such that $H=\bigoplus_{n \in \mathbb{N}} V_{n}$, where $V_{n}=\left[x_{i}\right]_{i \in D_{n}}$.

Define $G_{i}=\left\{x_{n}\right\}_{n \in D_{i}}$ and for each $j \in \mathbb{N}$, choose $\left\{y_{i}^{j}\right\}_{i \in \mathbb{N}}$ such that $y_{i}^{j}=j$ th element of $G_{i}$. Then for each $j,\left\{y_{i}^{j}\right\}$ is a Riesz basic sequence for $H$ and $\left\{x_{n}\right\}=\bigcup_{j}\left\{y_{i}^{j}\right\}$.

\section{ACKNOWLEDGEMENTS.}

The authors thank the referees for thoroughly reading the paper and for providing valuable comments and useful suggestions for the improvement of the paper. The research of first author is partially supported by the UGC (India) (Letter No.F.6-1(52)/2007 (MRP/Sc/NRCB)) and the research of third author is supported by the CSIR (India) (vide letter No. 09/172(0053)/2006EMR-I dated 19.07.06).

\section{REFERENCES}

[1] R. Balan, P. G. Casazza and D. Edidin, On signal reconstruction without phase, Appl. Comput. Harmon. Anal. 20 (2006), 345-356.

[2] J. J. Benedetto and M. Fickus, Finite normalized tight frames, Frames, Adv. Comput. Math. 18 (2003), 357-385.

[3] P. G. Casazza, The art of frame theory, Taiwanese J. Math. 4 (2000), 129-201.

[4] P. G. Casazza, Custom building finite frames, in: Wavelets, frames and operator theory, 61-86, Contemp. Math. 345, Amer. Math. Soc., Providence, RI, 2004.

[5] P. G. Casazza, O. Chirstensen, A. Linder and R. Vershynin, Frames and Feichtinger Conjecture, Proc. Amer. Math. Soc. 133 (2005), 1025-1033.

[6] P. G. Casazza and G. Kutyniok, Robustness of fusion frames under erasures of subspaces and of local frame vectors, Random transforms, geometry, and wavelets (New Orleans, LA, 2006), Contemp. Math., Amer. Math. Soc., Providence, RI (to appear).

[7] P. G. Casazza, G. Kutyniok, D. Speegle and J. C. Tremain, A decomposition theorem for frames and the Feichtinger conjucture, Proc. Amer. Math. Soc. 136 (2008), 20432053.

[8] P. G. Casazza, G. Kutyniok and S. Li, Fusion frames and distributed processing, Appl. Comput. Harmon. Anal. 25 (2008), 114-132.

[9] P. G. Casazza, G. Kutyniok, S. Li and C. J. Rozell, Modeling sensor networks with fusion frames. Wavelets XII (San Diego, CA, 2007), 67011M-1 - 67011M-11, SPIE Proc. 6701, SPIE, Bellingham, WA, 2007.

[10] O. Christensen, An Introduction to Frames and Riesz Bases, Birkhäuser Boston, Inc., Boston, MA, 2003.

[11] O. Christensen, Linear combinations of frames and frame packets, Z. Anal. Anwendungen 20 (2001), 805-815.

[12] I. Daubechies, A. Grossmann and Y. Meyer, Painless non-orthogonal expansions, J. Math. Phys. 27 (1986), 1271-1283. 
[13] R. J. Duffin and A. C. Schaeffer, A class of nonharmonic Fourier series, Trans. Amer. Math. Soc. 72 (1952), 341-366.

[14] S. J. Favier and R. A. Zalik, On the stability of frames and Riesz bases, Appl. Comput. Harmon. Anal. 2 (1995), 160-173.

[15] D. Gabor, Theory of communications, J. IEE (London) 93 (1946), 429-457.

[16] C. Heil and D. Walnut, Continuous and discrete wavelet transform, SIAM Rev. 31 (1989), 628-666.

[17] R. V. Kadison and I. M. Singer, Extensions of pure states, Amer. J. Math. 81 (1959), 383-400.

[18] G. Kutyniok, A. Pezeshki, A. R. Calderbank and T. Liu, Robust dimension reduction, fusion frames, and Grassmannian packings, preprint.

S. K. Kaushik

Department of Mathematics

Kirori Mal College,

University of Delhi

Delhi 110007

India

E-mail: shikk2003@yahoo.co.in

G. Singh

Department of Mathematics and Statistics,

University College of Science, M.L.S. University,

Udaipur (Rajasthan)

India

E-mail: Ghanshyamsrathore@yahoo.co.in

Virender

Department of Mathematics and Statistics,

University College of Science, M.L.S. University,

Udaipur (Rajasthan)

India

E-mail: virender57@yahoo.com

Received: 12.9.2007.

Revised: 2.1.2008. \& 29.1.2008. 